\title{
Integrated Pest Management of Guava, Citrus and Mango Fruitflies at Three Districts of Punjab
}

\author{
Muneer Abbas ${ }^{1 *}$, Dilbar Hussain ${ }^{2}$, Muhammad Saleem², Abdul Ghaffar ${ }^{2}$, \\ Sohail Abbas ${ }^{3}$, Niaz Hussain ${ }^{1}$ and Abdul Ghaffar ${ }^{1}$ \\ ${ }^{1}$ Arid Zone Research Institute, Bhakkar \\ ${ }^{2}$ Entomological Research Institute, Ayub Agricultural Research Institute, Faisalabad \\ ${ }^{3}$ Department of Entomology, University of Agriculture, Faisalabad
}

\begin{abstract}
A B S T R A C T
Fruit fly, Bactrocera zonata and Bactrocera dorsalis (Diptera: Tephritidae) has endangered the trade of Pakistani fruits in foreign markets. Use of IPM is best way to control fruit fly by avoiding harmful insecticidal spray application in order to save natural enemies and environment. Experiment was conducted in three guava, citrus and mango producing districts of Punjab i.e. Faisalabad, Sargodha, Toba Tek Singh with five treatments. Each experimental unit comprised of 1 ha area. Five treatments viz. $\mathrm{A}=$ sanitation, $\mathrm{B}=\mathrm{MAT}, \mathrm{C}=$ protein based baits, $\mathrm{D}=$ plant extracts and $\mathrm{E}=\mathrm{A}+\mathrm{B}+\mathrm{C}+\mathrm{D}$ were used during 2015-16. Data of \% infested fallen fruits, total flies/trap, total flies captured/year, $\%$ fruit punctures, pupal population, $\%$ fruit infestation and market value of fruits was collected at regular intervals. Results indicated that when all the components were applied in a combined way they gave significant reduction of fruit losses. As a result of continuous sanitation practices the fruit fall reduced to 10.24, 7.04, and 8.39 $\%$ in guava, citrus and mango orchards, respectively during following year in block E. Population peaks were May-July, July-September and July-August in guava, citrus and mango orchards with maximum population 69.06-70.36, 51.0-58.32 and 37.54-43.92/trap, respectively with TFC (total flies captured) reduced during $2^{\text {nd }}$ year. Plant extracts Azadirachta indica and Citrullus colocynthis proved better as they reduced pupal population 14.53 and $10.74,9.87$ and $2.85,7.20$ and $2.27 \%$ in guava, citrus and mango orchards, respectively. Similarly, reduced trend was found in \% fruit infestation by 19.41 and 10.29, 15.51 and $10.77,5.84$ and $4.80 \%$ in guava, citrus and mango orchards, respectively after $2^{\text {nd }}$ spray. Maximum $\%$ reduction of fruit punctures were found as $14.41,7.17$ and 7.32 in guava, citrus and mango orchards, respectively with combined application of all components. Market value (\$) of fruits was maximum in the block having complete IPM package with 15.60, 14.09 and $10.32 \%$ increased value of guava, citrus and mango orchards during following year.
\end{abstract}

Article Information
Received 15 January 2010
Revised 25 July 2019
Accepted 04 March 2020
Available online 26 March 2021
Authors' Contribution
MA conducted the research and wrote
the manuscript. DH designed the
experiments and conducted research.
MS and AG collected data. SA, NH
and AG reviewed the literature.
Key words
Fruitfly, IPM, Fruit orchards, Fruit
losses, Market value

Article Information

Revised 25 July 2019

Accepted 04 March 202

Available online 26 March 2021

Authors' Contribution

MA conducted the research and wrote (hesigned the eriments and conducted research. and $A G$ reviewed the literature.

Fruitfly, IPM, Fruit or
losses, Market value

\section{INTRODUCTION}

$\mathrm{F}$ ruit flies are damaging pests in the world, feeding and attacking most of the fruits and vegetables due to their very wide host range, high reproductive potential and adaptability to certain climates. Tephritids fruit flies are big threat to horticulture industry in Pakistan (Ekesi and Billah, 2007) because most of fruit flies are polyphagous attacking several fruits and vegetables including guava, citrus, mango, tomatoes, pepper, cucurbits etc and causing direct and indirect losses. Moreover residues of pesticides used against this pest are threat to export (Sarwar, 2013). Fruit flies are serious pest of guava fruit in Pakistan. Their damaging nature is a big hurdle for the promotion of export of fruits and vegetables by causing direct and indirect

\footnotetext{
* Corresponding author: m.abbas1902@gmail.com 0030-9923/2021/0003-0995 \$ 9.00/0
}

Copyright 2021 Zoological Society of Pakistan losses. Peach fruit fly, Bactrocera zonata (S) and Oriental fruit fly, Bactrocera dorsalis $(\mathrm{H})$ are most economically important fruit fly species in fruits (Sarwar, 2014). In Pakistan, the fruit fly complex may cause loses from 20$90 \%$ in different areas of the country. They cause heavy loses to fruits at farm level with approx. 200 million US dollars annually (Stonehouse et al., 2002). In Egypt annual losses of peach fruit fly were estimated 190 million $€$. These are most destructive in mangoes as it causes 50$85 \%$ loses and one can loose $100 \%$ of fruits (Nankinga et al., 2014). Female flies lay their eggs in the fruits and the maggots after emergence, eat the pulp leading to secondary infections with bacterial and fungal diseases (White and Elson-Harris, 1994). Integrated Pest Management (IPM) is the best way for proper management of fruit fly such as use of MAT (male annihilation technique), sanitation, protein based baits and plant extracts at regular intervals. MAT with methyl eugenol is a common method of management and a part of IPM (Afzal and Javed, 2001). Methyl eugenol 
is a plant component that is derived from essential oils of 200 plant species from 32 families. This disturbs male/ female ratio which consequently produce few progenies (Zaheeruddin, 2007). During last decade new powerful attractants have been developed to make mass trapping more economical (Epsky et al., 1999). This technique is successful for guava, mango and citrus (McQuate et al., 2005). In Spain about 30,000 ha of citrus were treated with mass trapping (Navarro-Llopis et al., 2008). More studies are needed for trap density optimization, shape of trap, pre-harvest placing time and dispensers covering entire growing season because efficacy of dispensers depends upon the climatic conditions. Sanitation, protein baits, plant extracts are more reliable ways to stop reproductive cycle without use of toxic chemicals. Protein baits containing hydrolyzed protein with amino acids or peptides $\mathrm{n}$ with amino acids or peptides and insecticide had been used to control several tephritids flies (Moreno et al., 2001; Vargas et al., 2001). In guava crop currently 5-7 sprays and in mango fruits 2-3 sprays are being applied after every 10150 days. Insecticides application against fruit flies are 10\% of total synthetic insecticides applied in Pakistan. Plant extracts are generally pest specific and relatively harmless to non-target organisms and environment. Azadirachta indica and Colocynthis colocynthis have repellent effects and used as biopesticide against $B$. zonata and $B$ dorsalis (Singh et al., 2007; Solangi et al., 2011). These act as antifeedent, repellent and egg laying deterrent. Derivatives of $P$. hysterophorus can be used to control different insects (Datta and Sexena, 2001). Costea and Tardif (2006) reported antifungal and insecticidal effects of Cascuta sp. Nerium oleander owns antibacterial (Derwic et al., 2010), antifungal and antioxidant activities (Mohadjerani, 2012; Ali et al., 2010).

Objective of the study was to develop a complete IPM package to break reproductive cycle of fruitflies without use of toxic chemicals by using MAT, sanitation, protein based spray and plant extracts alone or in a combination at regular intervals in guava, citrus and mango fruit orchards and to evaluate the effects of different components based on market value of fruits in comparison with conventional farmer's technology.

\section{MATERIALS AND METHODS}

Experiment was conducted in three different districts of Punjab i.e. Faisalabad, Sargodha, Toba Tek Singh during 2015-16 at farmer fields. Three orchards i.e. guava, citrus, mango in each district were selected for the experiment. Each district was considered as a replicate of experiment with six treatments. Each orchard comprised of 1 hectare area having five blocks viz. A, B, C, D and E (Table I). In block A only, sanitation was practiced. Average infested fallen fruits with fruit fly were counted on per plant basis on fortnightly intervals continuously for two years. These fruits were collected and buried at 10"-12" depth in the soil at regular intervals. After fruit burial soil was compressed firmly so that any larvae or adult cannot come out of soil. MAT was applied in block B which involved use of a male lure combined with an insecticide in order to reduce male population of fruit flies. This resulted mating of male population with female flies at low level. Fifteen pet bottle traps/ha were used, manufactured at home. Each trap was treated with $0.5 \mathrm{ml}$ of methyl eugenol (4-allyl-1, 2 dimethoxy benzene-carboxylate) + Spinosad insecticide (3:1) after every 14 days with the help of cotton wicks. Traps were hanged on trees at 1.5-2.0 m height above ground. Data was collected at fortnightly intervals. Adult flies were collected, counted from every trap. Meteorological data of all the districts was collected from Meteorological Department Lahore. Different plant extracts (block C) viz. Azadirachta indica, Parthenium hysterophorus, Cascuta campestris, Nerium oleander and Citrullus colocynthis were tested due to their repellency and larvicidal properties. Different plant parts were dried in oven at $60-70{ }^{\circ} \mathrm{C}$ and grinded separately with electic grinder. Each plant was extracted with analytical grade acetone in Soxhlet apparatus. The extracts obtained were stored at $4{ }^{\circ} \mathrm{C}$ in separate conical flask. One gram of each crude extract was dissolved in $10 \mathrm{ml}$ acetone. Thus, 100 $\mathrm{g}$ crude extracts of each plant was used for preparing 100 $\mathrm{ml}$ stock solution. Their effects were assessed by percent fruit infestation and pupal population below the canopy of trees before and 21 days after spray. Pupal population was assessed 6'-8' under guava, 8'-10' under citrus and $10^{\prime}-12$ ' under mango canopy by sieving the sand upto 6" depth. Application of protein hydrolyzate + malathion 50 EC (95:05) was made in block D at $1 \mathrm{~m}^{2}$ area of plants. Application of all these IPM treatments were combined in block E. Efficacy of all the treatments was assessed by percent fruit punctures, percent fruit infestation, infested fallen fruits and market value (\$) of fruits as compared to the orchards under farmers practices.

\section{Statistical analysis}

Mean population in all the traps was transformed (SQRT $(\mathrm{X}+0.5))$ to normalized the distribution in the trap evaluation. All means were separated using least significant difference (LSD) test at $P 0.05$ (SAS Institute, 2003). Further to measure goodness of fit, the values of coefficient of determination $\left(\mathrm{R}^{2}\right)$ were calculated by MS Excel. Data on fruit punctures, pupal population, fallen fruits, fruit infestation and fruit price was statistically analyzed by ANOVA using Statistica (Statsoft, 1998). 
Table I. Layout of five fruit orchards blocks each of 1 hectare.

\begin{tabular}{|c|c|c|c|c|}
\hline Block A & Block B & Block C & Block D & Block E \\
\hline$\times \times \times \times \times \times \times \times$ & $\times \times \times \times \times \times \mathbf{T} \times$ & $\times \times \times \times \times \times$ PE PE & $\times \times \times \times \times \mathbf{P H} \times \times$ & $\mathbf{T} \times \times \times \times \times \mathbf{P H} \times$ \\
\hline$\times \times \times \times \times \times \times \times$ & $\times \mathbf{T} \times \times \times \times \times \times$ & $\times \times \times \times$ PE PE $\times \times$ & $\times \times \mathbf{P H} \times \times \times \times \times$ & $\times \times \times \mathbf{P E} \times \times \times \mathrm{T}$ \\
\hline$\times \times \times \times \times \times \times \times$ & $\times \times \times \times \times \times \mathbf{T} \times$ & $\times \times$ PE PE $\times \times \times \times$ & $\times \times \times \times \times \mathbf{P H} \times \times$ & $\mathbf{T} \times \times \times \times \times \mathbf{P H} \times$ \\
\hline$\times \times \times \times \times \times \times \times$ & $\times \mathbf{T} \times \times \times \times \times \times$ & PE PE $\times \times \times \times \times \times$ & $\times \times \mathbf{P H} \times \times \times \times \times$ & $\times \times \times \mathbf{P E} \times \times \times \mathbf{T}$ \\
\hline$\times \times \times \times \times \times \times \times$ & $\times \times \times \times \times \times \mathbf{T} \times$ & $\times \times$ PE PE $x \times \times \times$ & $\times \times \times \times \times$ PH $\times \times$ & $\mathbf{T} \times \times \times \times \times \mathbf{P H} \times$ \\
\hline$\times \times \times \times \times \times \times \times$ & $\times \times \times \times \times \times \times \times$ & $\times \times \times \times$ PE PE $\times \times$ & $\times \times \mathbf{P H} \times \times \times \times \times$ & $\times \times \times \mathbf{P E} \times \times \times \times$ \\
\hline$\times \times \times \times \times \times \times \times$ & $\times \mathbf{T} \times \times \times \times \times \times$ & $\times \times \times \times \times \times$ PE PE & $\times \times \times \times \times \mathbf{P H} \times \times$ & $\mathbf{T} \times \times \times \times \times \mathbf{P H} \times$ \\
\hline$\times \times \times \times \times \times \times \times$ & $\times \times \times \times \times \times \times \times$ & $\times \times \times \times$ PE PE $\times \times$ & $\times \times \mathbf{P H} \times \times \times \times \times$ & $\times \times \times \mathbf{P E} \times \times \times \times$ \\
\hline$\times \times \times \times \times \times \times \times$ & $\times \times \times \times \times \times \mathbf{T} \times$ & $\times \times$ PE PE $\times \times \times \times$ & $\times \times \times \times \times \mathbf{P H} \times \times$ & $\mathbf{T} \times \times \times \times \times \mathbf{P H} \times$ \\
\hline$\times \times \times \times \times \times \times \times$ & $\times \times \times \times \times \times \times \times$ & PE PE $\times \times \times \times \times \times$ & $\times \times \mathbf{P H} \times \times \times \times \times$ & $\times \times \times \mathbf{P E} \times \times \times \times$ \\
\hline$\times \times \times \times \times \times \times \times$ & $\times \mathbf{T} \times \times \times \times \times \times$ & $x \times$ PE PE $x \times x \times$ & $\times \times \times \times \times \mathbf{P H} \times \times$ & $\mathbf{T} \times \times \times \times \times \mathbf{P H} \times$ \\
\hline$\times \times \times \times \times \times \times \times$ & $\times \times \times \times \times \times \times \times$ & $\times \times \times \times$ PE PE $\times \times$ & $\times \times \mathbf{P H} \times \times \times \times \times$ & $\times \times \times \mathbf{P E} \times \times \times \times$ \\
\hline$\times \times \times \times \times \times \times \times$ & $\times \times \times \times \times \times \mathbf{T} \times$ & $\times \times \times \times \times \times$ PE PE & $\times \times \times \times \times$ PH $\times \times$ & $\mathbf{T} \times \times \times \times \times \mathbf{P H} \times$ \\
\hline$\times \times \times \times \times \times \times \times$ & $\times \times \times \times \times \times \times \times$ & $\times \times \times \times$ PE PE $\times \times$ & $\times \times \mathbf{P H} \times \times \times \times \times$ & $\times \times \times \mathbf{P E} \times \times \times \times$ \\
\hline$\times \times \times \times \times \times \times \times$ & $\times \mathbf{T} \times \times \times \times \times \times$ & $x \times$ PE PE $x \times x \times$ & $\times \times \times \times \times \mathbf{P H} \times \times$ & $\mathbf{T} \times \times \times \times \times \mathbf{P H} \times$ \\
\hline$\times \times \times \times \times \times \times \times$ & $\times \times \times \times \times \times \times \times$ & PE PE $\times \times \times \times \times \times$ & $\times \times \mathbf{P H} \times \times \times \times \times$ & $\times \times \times \mathbf{P E} \times \times \times \times$ \\
\hline$\times \times \times \times \times \times \times \times$ & $\times \times \times \times \times \times \mathbf{T} \times$ & $\times \times$ PE PE $\times \times \times \times$ & $\times \times \times \times \times \mathbf{P H} \times \times$ & $\mathbf{T} \times \times \times \times \times \mathbf{P H} \times$ \\
\hline$\times \times \times \times \times \times \times \times$ & $\times \times \times \times \times \times \times \times$ & $\times \times \times \times$ PE PE $\times \times$ & $\times \times \mathbf{P H} \times \times \times \times \times$ & $\times \times \times \mathbf{P E} \times \times \times \times$ \\
\hline$\times \times \times \times \times \times \times \times$ & $\times \mathbf{T} \times \times \times \times \times \times$ & $\times \times \times \times \times \times$ PE PE & $\times \times \times \times \times \mathbf{P H} \times \times$ & $\mathbf{T} \times \times \times \times \times \mathbf{P H} \times$ \\
\hline$\times \times \times \times \times \times \times \times$ & $\times \times \times \times \times \times \mathbf{T} \times$ & $\times \times \times \times$ PE PE $\times \times$ & $\times \times \mathbf{P H} \times \times \times \times \times$ & $\times \times \times \mathbf{P E} \times \times \times \mathrm{T}$ \\
\hline$\times \times \times \times \times \times \times \times$ & $\times \mathbf{T} \times \times \times \times \times \times$ & $\times \times$ PE PE $\times \times \times \times$ & $\times \times \times \times \times \mathbf{P H} \times \times$ & $\mathbf{T} \times \times \times \times \times \mathbf{P H} \times$ \\
\hline$\times \times \times \times \times \times \times$ & $\times \times \times \times \times \times \mathbf{T} \times$ & PE PE $\times \times \times \times \times \times$ & $\times \times \mathbf{P H} \times \times \times \times \times$ & $\times \times \times \mathbf{P E} \times \times \times \mathrm{T}$ \\
\hline
\end{tabular}

T, Traps; ${ }^{\text {PH }}$, Protein based spray; ${ }^{\text {PE }}$, Plant extract spray.

\section{RESULTS AND DISCUSSION}

During the $1^{\text {st }}$ year of study population attraction by the MAT technique was more aggressive as compared to the $2^{\text {nd }}$ year. However, the population trends during both years were not significantly different from each other in all fruit orchards. Population peaks in guava orchard were found during the months of May-July with maximum captures 70.36 and 69.06 adults/trap, TFC were 1884 and 1697 adults/season/acre during year 2015-16, respectively. Trend line $\left(\mathrm{y}=-0.4891 \mathrm{x}+30.993, \mathrm{R}^{2}=0.02\right)$ shows negative correlation of the regression line with population trends of both years. Population peaks in citrus orchard were found during the months of July-September with maximum captures 58.32 and 51.00 adults/trap, TFC were 1935 and 1743adults/season/acre during year 201516 , respectively. Trend line $\left(\mathrm{y}=1.9183 \mathrm{x}+1.5738, \mathrm{R}^{2}=\right.$ 0.3665 ) shows positive correlation with most fit regression line. In mango orchard population peaks were found during the months of July-August with maximum captures 37.54 and 43.92 adults/trap. Total flies captured (TFC) were 1646 and 1414 adults/season/acre during year 201516 , respectively. Trend line $\left(\mathrm{y}=0.7643 \mathrm{x}+3.1153, \mathrm{R}^{2}=\right.$ 0.1924 ) shows positive correlation with most fit regression line of both years population trends as shown in Figure 1. There was no significant deference of meteorological factors among two years. To reduce the extent of damage average infested fruits by fruit fly (Fig. 2) were estimated on plant basis. Ratio of fallen fruits was low in 2016 as compared to 2015. In guava orchard maximum 10.97 and 10.65 fallen fruits/plant were recorded during the month of April 2015-16, respectively. Level of infestation in guava was much higher as compared to the other fruit orchards. In citrus orchard maximum 3.36 and 2.98 fallen fruits/ plant were recorded during the month of December 201516 , respectively. However, in mango orchard maximum 6.74 and 6.90 fallen fruits/ plant were recorded during the month of July 2015-16, respectively. Fallen fruits were buried (Table II) at regular intervals. However total fallen fruits in all the IPM blocks were varied depending upon their efficacy. Fallen fruits in all treatments were higher except in the block having all the components 
applied in combined way. This block depicted minimum fruit falling with maximum reduction in fruit falling in the following year. During $2016 \%$ reduction in fruit fall was $10.24,7.04$ and 8.39 /ha in guava, citrus and mango orchards, respectively as compared to 2015. While all the other IPM blocks had enhanced fruit falling with minimum reduction in fruit fall. Similar type of response was noticed when calculated their effects on percent fruit punctures by the fruit fly (Table III). Range of fruit single punctures was 13.75-49.51, 2.67-13.64 and 3.25-13.75 $\%$ in guava, citrus and mango fruits, respectively. Fruit punctures in all the IPM blocks were higher except in complete IPM block E having minimum fruit punctures with maximum puncture reduction in the following year. As it showed 10.41, 7.17 and $7.32 \%$ reduced punctures in guava, citrus and mango orchards, respectively in 2016 as compared to the previous year. Different plant extracts i.e. A. indica, P. hysterophorus, C. campestris, $N$. oleander and $C$. colocynthis were tested to check the pupal population under the tree canopy and percent fruit infestation. Range of pretreatment pupal population (Table IV) was $17.36-32.56,4.52-8.64$ and $4.68-8.73$ in guava, citrus and mango orchards, respectively. After 21 days of $1^{\text {st }}$ spray A. indica, $P$. hysterophorus and C. colocynthis reduced pupal population and \% fruit infestation by 11.83 and $15.35,9.03$ and $10.79,7.4$ and 11.48 in guava orchard. $A$. indica reduced pupal population and $\%$ fruit infestation by 6.44 and 8.01 in citrus orchard, 5.5 and 3.52 in mango orchard, respectively. Range of pre treatment pupal population before $2^{\text {nd }}$ application (Table IV) was 13.69-23.98, 4.79-8.75 and 4.86-8.36 in guava, citrus and mango orchards, respectively. After 21 days of $2^{\text {nd }}$ spray A. indica, P. hysterophorus and C. colocynthis reduced pupal population and \% fruit infestation by 14.53 and $19.41,13.95$ and $12.95,10.74$ and 10.29 in guava orchard, 9.47 and 15.51 in citrus orchard by $A$. indica, 7.2 and 5.84 in mango orchard by $A$. indica, respectively. Application of protein based protein hydrolyzate + malathion $50 \mathrm{EC}$ produced non-significant reduction in \% fruit punctures and fallen fruits (Tables II and III). It reduced 1.97, 0.04 and $0.15 \%$ fruit punctures in guava, citrus and mango orchards, respectively with $1.09,2.51 \%$ reduced fruit fall in guava and citrus orchard. However increased fruit fall by $3.98 \%$ in mango orchard. Depending upon the efficacy of different IPM modules market value of fruits was computed based on the fruit quality and infestation level (Table V). Maximum market value was obtained from block E having complete IPM components withoutcome 2184, 2200, $2520 \$ /$ ha in guava, citrus and mango orchard, respectively during $1^{\text {st }}$ year. Due to continuous application of IPM module income got 15.60, 14.09 and $10.32 \%$ increase in guava, citrus and mango orchard, respectively. When compared with farmer fields they got $1890,1825,2330 \$ /$ ha from guava, citrus and mango orchards, respectively during $1^{\text {st }}$ year with only $1.59 \%$ increase in guava orchard while 0.27 and $0.86 \%$ decreased income from citrus and guava orchards. Where increase is very nominal as compared to the IPM blocks.

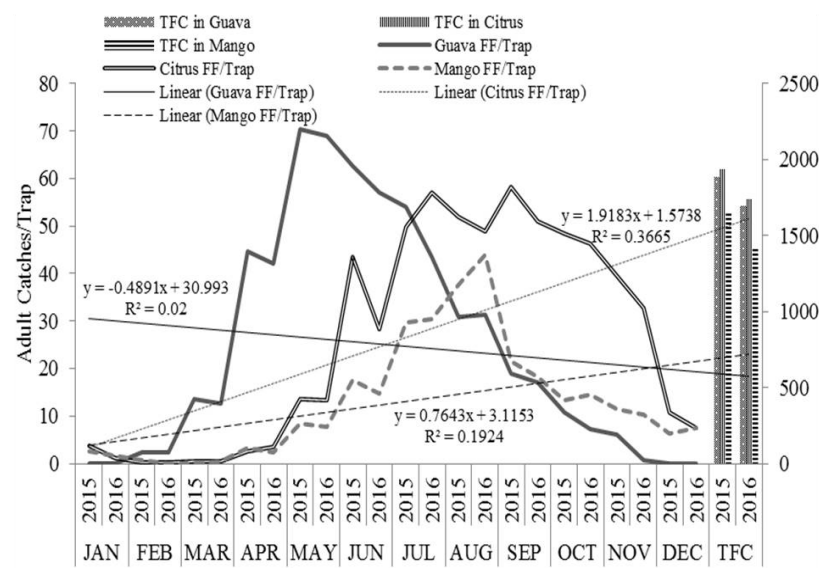

Fig. 1. Total fruit flies captured with average/ trap by male annihilation technique in different fruit orchards during 2015-16.

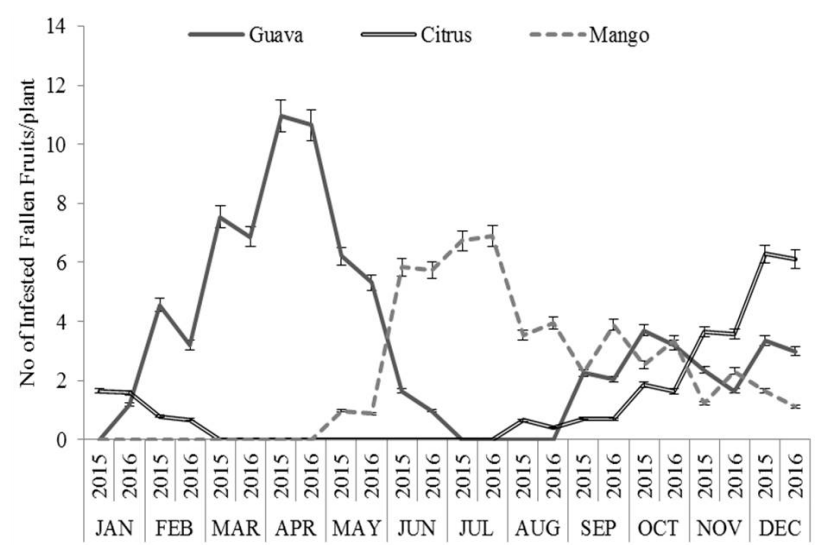

Fig. 2. Average number of infested fallen fruits/ plant.

Population peaks of fruitflies were different in all fruit orchards. Peaks were May-July, July-September and JulyAugust in guava, citrus and Mango orchards through MAT. Several scientists worked on the population fluctuation of fruitflies by male attractants. They reported population peaks on March, April, May and June (Sarada et al., 2001; Latif and Abdullah, 2005; Selvaraj et al., 2006; Boopathi et al., 2012; Boopathi, 2013) which are in agreement with our findings in guava and citrus orchards as it may be due to the availability of ample guava fruit in our peaks and ideal temperature for the activity. But the reported peaks were not correlating in mango orchard. But Draz et al. (2016) 
Table II. Total number of buried fallen fruits/ha/year in different fruit orchards infested with fruitfly during 2015 16.

\begin{tabular}{|c|c|c|c|c|c|c|c|c|c|c|}
\hline \multirow[t]{2}{*}{ Block } & \multirow[t]{2}{*}{ IPM treatments } & \multicolumn{2}{|c|}{ Guava } & \multirow{2}{*}{$\begin{array}{c}\% \\
\text { Change }\end{array}$} & \multicolumn{2}{|c|}{ Citrus } & \multirow{2}{*}{$\begin{array}{c}\% \\
\text { Change }\end{array}$} & \multicolumn{2}{|c|}{ Mango } & \multirow{2}{*}{$\begin{array}{c}\% \\
\text { Change }\end{array}$} \\
\hline & & 2015 & 2016 & & 2015 & 2016 & & 2015 & 2016 & \\
\hline A & Sanitation & 3325 & 3100 & $-6.77^{b}$ & 856 & 852 & $-0.47^{c}$ & 635 & 618 & $-2.68^{b}$ \\
\hline B & Male annihilation technique & 3536 & 3320 & $-6.11^{b}$ & 1136 & 1145 & $0.79^{\mathrm{e}}$ & 710 & 712 & $0.28^{\mathrm{c}}$ \\
\hline $\mathrm{C}$ & Plant Extracts & 3025 & 2880 & $-4.79^{c}$ & 986 & 954 & $-3.25^{\mathrm{b}}$ & 587 & 582 & $-0.85^{b}$ \\
\hline $\mathrm{D}$ & Protein hydrolyzate +Malathion 50 EC & 2759 & 2729 & $-1.09^{\mathrm{d}}$ & 1036 & 1010 & $-2.51^{b}$ & 854 & 888 & $3.98^{\mathrm{d}}$ \\
\hline $\mathrm{E}$ & Complete IPM package (A-D) & 1954 & 1754 & $-10.24^{\mathrm{a}}$ & 639 & 594 & $-7.04^{\mathrm{a}}$ & 274 & 251 & $-8.39^{\mathrm{a}}$ \\
\hline $\mathrm{F}$ & Farmer Practice & 3352 & 3525 & $5.16^{\mathrm{e}}$ & 1459 & 1460 & $0.07^{\mathrm{d}}$ & 1159 & 1210 & $4.40^{\mathrm{d}}$ \\
\hline
\end{tabular}

Table III. Effect of different IPM components on fruit punctures (\%) in different fruit orchards.

\begin{tabular}{|c|c|c|c|c|c|c|c|c|c|c|}
\hline \multirow[t]{2}{*}{ Block } & \multirow[t]{2}{*}{ IPM treatments } & \multicolumn{2}{|c|}{ Guava } & \multirow[t]{2}{*}{ Change } & \multicolumn{2}{|c|}{ Citrus } & \multirow[t]{2}{*}{ Change } & \multicolumn{2}{|c|}{ Mango } & \multirow[t]{2}{*}{ Change } \\
\hline & & 2015 & 2016 & & 2015 & 2016 & & 2015 & 2016 & \\
\hline A & Sanitation & 36.21 & 31.72 & $-4.49^{b}$ & 10.65 & 9.64 & $-1.01^{b}$ & 8.64 & 7.49 & $-1.15^{b}$ \\
\hline B & Male annihilation technique & 28.69 & 24.68 & $-4.01^{b}$ & 7.64 & 7.11 & $-0.53^{b}$ & 12.64 & 11.42 & $-1.22^{b}$ \\
\hline $\mathrm{C}$ & Plant extracts & 38.64 & 35.64 & $-3.00^{\mathrm{b}}$ & 11.42 & 10.56 & $-0.86^{\mathrm{b}}$ & 11.64 & 11.74 & $0.10^{\mathrm{b}}$ \\
\hline $\mathrm{D}$ & Protein hydrolyzate +Malathion 50 EC & 48.31 & 46.34 & $-1.97^{\mathrm{b}}$ & 13.64 & 13.60 & $-0.04^{b}$ & 13.75 & 13.60 & $-0.15^{b}$ \\
\hline E & Complete IPM package (A-D) & 24.16 & 13.75 & $-10.41^{\mathrm{a}}$ & 9.84 & 2.67 & $-7.17^{\mathrm{a}}$ & 9.67 & 2.35 & $-7.32^{\mathrm{a}}$ \\
\hline $\mathrm{F}$ & Farmer practice & 49.51 & 48.81 & $-0.70^{\mathrm{b}}$ & 11.64 & 12.57 & $0.93^{\mathrm{b}}$ & 10.48 & 11.21 & $0.73^{\mathrm{b}}$ \\
\hline
\end{tabular}

Table IV. Effect of different plant extracts on pupal population and fruit infestation by fruitfly in different fruit orchards.

\begin{tabular}{|c|c|c|c|c|c|c|c|c|c|c|c|c|c|}
\hline \multirow[t]{2}{*}{$\begin{array}{l}\text { Spray } \\
\text { (No) }\end{array}$} & \multirow[t]{2}{*}{$\begin{array}{l}\text { Treatments/ } \\
\text { plant extracts }\end{array}$} & \multicolumn{3}{|c|}{$\begin{array}{c}\text { Pre treatment } \\
\text { pupal population }(\mathrm{No})\end{array}$} & \multicolumn{3}{|c|}{$\begin{array}{l}\% \text { change in pupal } \\
\text { population } 21 \text { DAS }\end{array}$} & \multicolumn{3}{|c|}{$\begin{array}{l}\text { Pre treatment fruit } \\
\text { infestation }(\%)\end{array}$} & \multicolumn{3}{|c|}{$\begin{array}{l}\% \text { change in fruit } \\
\text { infestation } 21 \text { DAS }\end{array}$} \\
\hline & & $\mathbf{A}$ & B & $\mathbf{C}$ & $\mathbf{A}$ & B & $\mathbf{C}$ & $\mathbf{A}$ & B & $\mathbf{C}$ & $\mathbf{A}$ & B & $\mathbf{C}$ \\
\hline \multirow[t]{8}{*}{$1^{\text {st }}$} & A. indica & 25.36 & 6.21 & 5.27 & -11.83 & -6.44 & -5.50 & 58.62 & 6.24 & 4.26 & -15.35 & -8.01 & -3.52 \\
\hline & P. hysterophorus & 32.56 & 8.64 & 4.68 & -9.03 & 1.27 & 6.20 & 46.36 & 10.25 & 5.81 & -10.79 & 13.37 & 2.75 \\
\hline & C. campestris & 19.45 & 5.23 & 7.24 & -1.23 & 5.35 & 3.73 & 64.95 & 9.54 & 3.69 & -3.34 & 1.78 & 7.59 \\
\hline & N. oleander & 17.36 & 8.41 & 6.91 & 2.88 & -2.38 & -2.17 & 39.68 & 7.36 & 5.72 & -9.93 & -1.90 & -0.52 \\
\hline & C. colocynthis & 21.36 & 7.49 & 8.73 & -7.40 & -1.74 & -0.46 & 44.78 & 14.25 & 4.64 & -11.48 & -4.35 & -1.29 \\
\hline & Check & 28.65 & 4.52 & 5.46 & 3.60 & 5.97 & 7.88 & 49.16 & 6.58 & 6.74 & 5.15 & 14.59 & 8.16 \\
\hline & P Value & & & & $\mathrm{P}<0.048$ & $\mathrm{P}>0.05$ & $\mathrm{P}>0.05$ & & & & $\mathrm{P}<0.040$ & $\mathrm{P}>0.05$ & $\mathrm{P}>0.05$ \\
\hline & CV (\%) & & & & 16.34 & 12.65 & 20.84 & & & & 23.65 & 18.64 & 11.54 \\
\hline \multirow[t]{8}{*}{$2^{\text {nd }}$} & A. indica & 17.69 & 5.49 & 4.86 & -14.53 & -9.47 & -7.20 & 47.91 & 5.74 & 4.11 & -19.41 & -15.51 & -5.84 \\
\hline & P. hysterophorus & 21.65 & 8.75 & 4.97 & -13.95 & 5.26 & 3.42 & 52.43 & 11.62 & 5.97 & -12.95 & 0.77 & -1.34 \\
\hline & C. campestris & 23.98 & 5.51 & 7.51 & -6.76 & 7.62 & 4.26 & 39.76 & 9.71 & 3.97 & -3.97 & -1.96 & 4.79 \\
\hline & N. oleander & 15.75 & 8.21 & 6.76 & -6.60 & 4.75 & -3.55 & 40.52 & 7.22 & 5.69 & -7.18 & -9.83 & -1.76 \\
\hline & C. colocynthis & 13.69 & 7.36 & 8.36 & -10.74 & -2.85 & -2.27 & 36.16 & 12.63 & 4.58 & -10.29 & -10.77 & -4.80 \\
\hline & Check & 19.65 & 4.79 & 5.89 & 2.34 & 10.86 & 7.13 & 58.36 & 7.54 & 7.29 & 9.10 & 14.99 & 8.23 \\
\hline & P Value & & & & $\mathrm{P}<0.035$ & $\mathrm{P}>0.05$ & $\mathrm{P}>0.05$ & & & & $\mathrm{P}<0.021$ & $\mathrm{P}>0.05$ & $\mathrm{P}>0.05$ \\
\hline & CV (\%) & & & & 9.57 & 13.68 & 14.68 & & & & 19.68 & 13.59 & 8.57 \\
\hline
\end{tabular}

*A, Guava orchard; B, Citrus orchard; C, Mango orchard; DAS, Days after spray. 
Table V. Economic output (\$/ha) of different fruit orchards by using different IPM components.

\begin{tabular}{|c|c|c|c|c|c|c|c|c|c|c|}
\hline \multirow[t]{2}{*}{ Block } & \multirow[t]{2}{*}{ IPM treatments } & \multicolumn{2}{|c|}{ Guava } & \multirow{2}{*}{$\begin{array}{l}\% \\
\text { Change }\end{array}$} & \multicolumn{2}{|c|}{ Citrus } & \multirow{2}{*}{$\begin{array}{l}\% \\
\text { Change }\end{array}$} & \multicolumn{2}{|c|}{ Mango } & \multirow{2}{*}{$\begin{array}{l}\% \\
\text { Change }\end{array}$} \\
\hline & & 2015 & 2016 & & 2015 & 2016 & & 2015 & 2016 & \\
\hline A & Sanitation & 1950 & 2010 & $3.08^{\mathrm{b}}$ & 2070 & 2170 & $4.83^{\mathrm{b}}$ & 2350 & 2450 & $4.26^{\mathrm{b}}$ \\
\hline B & Male annihilation technique & 2060 & 2200 & $6.80^{\mathrm{b}}$ & 2150 & 2290 & $6.51^{\mathrm{b}}$ & 2180 & 2290 & $5.05^{\mathrm{b}}$ \\
\hline $\mathrm{C}$ & Plant extracts & 2040 & 2100 & $2.94^{\mathrm{b}}$ & 2040 & 2110 & $3.43^{\mathrm{b}}$ & 2470 & 2510 & $1.62^{\mathrm{b}}$ \\
\hline $\mathrm{D}$ & Protein hydrolyzate+Malathion 50 EC & 1700 & 1810 & $6.47^{\mathrm{b}}$ & 1830 & 1900 & $3.83^{\mathrm{b}}$ & 2250 & 2370 & $5.33^{\mathrm{b}}$ \\
\hline $\mathrm{E}$ & Complete IPM package(A-D) & 2180 & 2520 & $15.60^{\mathrm{a}}$ & 2200 & 2510 & $14.09^{\mathrm{a}}$ & 2520 & 2780 & $10.32^{\mathrm{a}}$ \\
\hline $\mathrm{F}$ & Farmer practice & 1890 & 1920 & $1.59^{\mathrm{b}}$ & 1825 & 1820 & $-0.27^{\mathrm{c}}$ & 2330 & 2310 & $-0.86^{b}$ \\
\hline
\end{tabular}

reported maximum peaks in the month of August, October, November and December. The lowest no of catches were recorded from December-March showing no significance population. Agarwal and Kumar (1999) and Abdel-Galil et al. (2010) also recorded peaks June, July and September of Bactrocera spp with the ripening season of citrus, mango and guava. Ravikumar (2005) found two peaks i.e. $1^{\text {st }}$ week of July and $3^{\text {rd }}$ week of November with population 132.67 and 37.67/trap, respectively in guava and mango orchard. This study is quite in accordance with our results where maximum population in guava and mango orchards were $69.06-70.36$ and 37.54-43.92/trap, respectively. While in citrus orchard average population was 51-58.32/ trap which confirmed by Jemaa et al. (2010) who captured 63.68 and 67.77 fruit flies in two orchards of mandarin and washington, respectively. Population infestation levels can be reduced by applying other components of IPM such as sanitation, protein based spray, and plant extracts. Excessive fruit fall 10.65-10.97/plant was found in guava orchard as compared to citrus and mango orchards having 2.98-3.36 and 6.47-6.90, respectively. Removal of these infested fruits is very much important to reduce fruit fly population levels. Disposal of fallen fruits was carried out at regular intervals. Similar strategy was adopted by (Dowell et al., 2000). Tephritids larvae always pupate under the fruit/soil. Sanitation significantly reduces fruit fly population in the ecosystem (Panday, 2004). But this practice is not sufficient to eradicate fruit fly population completely (Klungness, 2005). Keeping in mind continuous burial of fruits was carried out. Total buried fallen fruits infested with fruit fly were 14599, 4552, 3060/ha/year in guava, citrus and mango orchards, respectively in five blocks of IPM. This practice not only reduced the application of toxic chemicals (Purcell et al., 1994) but also breaks down the life cycle of fly to reduce its infestation in the next years. During 2016 fruit fall was significantly reduces as $6.77,0.47$ and $2.68 \%$ in guava, citrus and mango orchards, respectively. Pinero et al. (2009) reported significantly more female fruit flies were captured in experimental plots that were categorized as having poor sanitation in comparison with good sanitation plots. There were no extensive studies of plant extracts application on fruit fly were found. Few studies on $A$. indica and $C$. colocynthis were found in literature. A. indica reduced fruit infestation by $15.35,8.01,3.52 \%$ after $1^{\text {st }}$ spray and $19.41,15.51,5.84 \%$ after $2^{\text {nd }}$ spray in guava, citrus and mango orchards, respectively. Infestation levels in A. indica and $P$. hysterophorus were 47.91 and $52.43 \%$ after $2^{\text {nd }}$ application. These findings were confirmed by Ali et al. (2011) who reported 41.92 and $45.95 \%$ fruit infestation in $A$. indica and P. hysterophorus treated plots. Dhilion et al. (2005), Oke (2008), Masood et al. (2009), Mahfuza et al. (2007) confirmed that $A$. indica can easily control populations of $B$. cucurbitae and $B$. dorsalis. C. colocynthis overall suppressed egg laying of B. zonata with $34.55 \%$ repellency and caused mortality against adults of Lipaphis erysimi (Soam et al., 2013). Some extracts in the study were used for the $1^{\text {st }}$ time on fruitfly but their toxicity already been reported on other insects. Khan and Omar (2015) found N. oleander and $P$. hysterophorus efficient to formulate commercial products. Raveen et al. (2017) evaluated highest larvicidal activity of $N$. oleander against mosquito species. Several other scientists Komalamisra et al. (2005), Raveen et al. (2014), El-Akhal et al. (2015), Ali et al. (2008) also confirmed larvicidal and insecticidal activity of $N$. Oleander. Effects of these treatments were assessed through fruit punctures. Percent single point punctures in citrus were 2.67-13.64 in different IPM blocks. Amos et al. (2016) found similar results in citrus having 14-16\% single point attack. But the findings of Jemaa et al. (2010) not in agreement with our findings as he reported higher percentage of punctured fruits in citrus. However, the fruit puncture study in guava and mango has been reported before.

\section{CONCLUSION}

Best way to minimize the threat of fruitflies to the horticultural industry is to adopt all the IPM components 
in a combined way because different components alone cannot be so effective. Continuous practices are needed to minimize fruitfly population and infestation with time. Such as use of methyl eugenol traps, burial of infested fruits, application of protein based baits and plant extracts at regular intervals. Among plant extracts $A$. indica and C. colocynthis proved to best if they are applied twice in the peak activity periods of fruitfly. However other components should be monitored at fortnightly intervals to get significant reduction in population level of fruitfly. This not only helpful in judicious use of insecticide but also protects natural enemies and environment from harmful effects.

\section{Statement of conflict of inerest}

The authors have declared no conflict of interest.

\section{REFERENCES}

Abdel-Galil, F.A., Amro, M.A., Abdel-Moniem, A.S.H. and El-Fandary, O.O., 2010. Population fluctuations and interspecific competition between Tephritid flies attacking fruit crops in the New Valley oases, Egypt. Arch. Phytopathol. Pl. Protect., 43: 647 659. https://doi.org/10.1080/03235400802021272

Afzal, M. and Javed, H., 2001. Evaluation of soaked wooden killer blocks for male annihilation (MA) on fruit fly Bactrocera Spp. (Diptera: Tephritidae). Online J. biol. Sci., 1: 577-579. https://doi. org/10.3923/jbs.2001.577.579

Agarwal, M.L. and Kumar, P., 1999. Effect of weather parameters on population dynamics of peach fruit fly, Bactrocera zonata (S). Entomology, 24: 81-84.

Ali, H., Ahmad, S., Hassan, G., Amin, A., Hussain, Z. and Naeem M., 2011. Bioefficacy of different plant extracts against melon fruit fly in bitter gourd. Pak. J. Weed Sci. Res., 17: 143-149.

Ali, H.F.M., El-Ella, F.M.A. and Nasr N.F., 2010. Screening of chemical analysis, antioxidant, antimicrobial and antitumor activity of essential oil of oleander (Nerium oleander) flower. Int. J. biol. Chem., 4: 190-202. https://doi.org/10.3923/ ijbc.2010.190.202

Ali, S.S., Ali, S., Munir, S. and Riaz, T., 2008. Insecticidal and bactericidal effects of ethanolic leaf extract of common oleander, Nerium oleander. Punjab Univ. J. Zool., 23: 81-90.

Amos, O.A., Gabriel, O.O., Olufisayo, I. and Yemisi, A.O., 2016. Effects of field attack by Ceratitis capitata W. (Diptera: Tephritidae) on the morphology and nutritional quality fresh fruit of Citrus sinensis L. African J. agric. Res., 11: 967-
973. https://doi.org/10.5897/AJAR2014.9232

Boopathi, T., 2013. Population dynamics of fruit flies, Bactrocera spp. in tomato, Lycopersicon esculentum M. J. Eco-friend. Agric., 8: 181-183.

Boopathi, T., Pathak, K.A. and Sithanantham, S., 2012. Exploratory bio-ecology studies on incidence of fruit flies in Mizoram. In: Proceedings of National Symposium on Plant Protection, Indian Institute of Horticultural Research Hess araghatta Lake P. O. Bengaluru-560089, Karanataka, India. pp. 66.

Costea, M. and Tardif, F.J., 2006. The biology of Canadian weeds. 133. Cuscuta campestris Yuncker. Canada J. Pl. Sci., 86: 293-316. https://doi. org/10.4141/P04-077

Datta, S. and Saxena, D.B., 2001. Pesticidal properties of Parthenin (Parthenium hysterophorus L.) and related compounds. Pest Manage. Sci., 57: 95-101. https://doi.org/10.1002/15264998(200101)57:1<95::AID-PS248>3.0.CO;2-J

Derwic, E., Benziane, Z. and Boukir, A., 2010. Antibacterial activity and chemical composition of the essential oil from flowers of Nerium oleander. J. Environ. Agric. Fd. Chem., 9: 1074-1084.

Dhillon, M.K., Singh, R., Naresh, J.S. and Sharma, H.C., 2005. The melon fruit fly, Bactrocera cucurbitae: A review of its biology and management. J. Insect Sci., 5: 1-16. https://doi.org/10.1093/jis/5.1.40

Dowell, R.V., Siddiqui, I.A., Meyer, F. and Spoungy, E.L., 2000. Mediterranean fruit fly preventative release program in southern California. In: Area wide control of fruit flies and other insect pests (ed. Keng-Hong). Sinaran Bros., Sdn. Bhd., Penang, Malaysia, pp. 369-375.

Draz, K.A., Reda M., Tabikhal, M.A., El-Aw1, ElGendy, I.R. and Darwish, H.F., 2016. Population activity of peach fruit fly Bactrocera zonata (S) (Diptera: Tephiritidae) at fruits orchards in Kafer El-Shikh Governorate, Egypt. Arthropods, 5: 2843.

Ekesi, S. and Billah, M.K., 2007. A field guide to the management of economically important tephritid fruit flies in Africa. ICIPE Science Press, Nairobi, Kenya.

El-Akhal F., Guemmouh, R., Zoubi, Y.E. and Lalami, A.E.O., 2015. Larvicidal activity of Nerium oleander against larvae West Nile vector mosquito Culex pipiens (Diptera: Culicidae). J. parasitol. Res., 1-5. https://doi.org/10.1155/2015/943060

Epsky, N.D., Hendrichs, J., Katsoyannos, B.I., Vasquez, L.A., Ros, J.P., Zumreoglu, A., Pereira, R., Bakri, A., Seewooruthun, S.I. and Heath, R.R., 1999. Field evaluation of female-targeted trapping systems for 
Ceratitiscapitata (Diptera: Tephritidae) in seven countries. J. econ. Ent., 92: 156-164. https://doi. org/10.1093/jee/92.1.156

Jemaa, J.M.B., Bachrouch, O., El Allimi and Dhouibi, M.H., 2010. Field evaluation of Mediterranean fruit fly mass trapping with Tripack ${ }^{\circledR}$ as alternative to malathion bait-spraying in citrus orchards. Span. J. agric. Res., 8: 400-408. https://doi.org/10.5424/ sjar/2010082-1187

Khan, I. and Qamar, A., 2015. Comparative bioefficacy of selected plant extracts and some commercial biopesticides against important household pest, Periplaneta americana. J. Ent. Zool. Stud., 3: 219224.

Klungness, L.M., Eric, B.J., Mau, R.F.L., Roger, Vargas, Sugano, J.S. and Fujitani, E., 2005. New sanitation techniques for controlling Tephritid fruit flies (Diptera: Tephritidae) in Hawaii. J. appl. Sci. environ. Mgt., 9: 5-14. https://doi.org/10.4314/ jasem.v9i2.17284

Komalamisra, N., Trongtokit, Y., Rongsriyam, Y. and Apiwathnasorn, C., 2005. Screening for larvicidal activity in some Thai plants against four mosquito vector species. Southeast Asian J. trop. Med. Publ. Hlth., 36: 1412-1422.

Latif, A. and Abdullah K., 2005. Population dynamics and management of Batrocera sp. (Diptera: Tephritidae) infesting mango. Pak. J. Ent., 27: 234-238.

Mahfuza, K., Aftab, M.H. and Saidu, M.I., 2007. Effect of Neem leaf dust and commercial formulation of a Neem compound on the longevity, fecundity and ovarian development of melon fly, Bactrocera cucurbitae (C) and oriental fruit fly, Bactrocera dorsalis (H) (Diptera: Tephritidae). Pak. J. biol. Sci., 10: 3656-3661. https://doi.org/10.3923/ pjbs.2007.3656.3661

Masood, K.K., Rashid, M.M.U. and Abdullah, K., 2009. Effect of Neem derivatives on infestation, settling and oviposition of melon fruit fly (Bactrocera cucurbitae C.) (Tephritidae: Dipetra). Pak. J. Ent., 31: 11-15.

Mcquate, G.T., Sylva, C.D. and Jang, E.B., 2005. Mediterranean fruit fly (Dip., Tephritidae) suppression in persimmon through bait sprays in adjacent coffee planting. J. appl. Ent., 129: 110-117. https://doi.org/10.1111/j.1439-0418.2005.00942.x

Mohadjerani, 2012. Antioxidant activity and total phenolic content of Nerium oleander L. grown in north of Iran. Iranian J. pharmaceut. Res., 11: 11211126.

Moreno, D.S., Celedonio, H., Mangan, R.L., Zavala, J.L. and Montoya, P., 2001. Field evaluation of a phototoxic dye, phloxine B, against three species of fruit bies. J. econ. Ent., 94: 1419-1427. https://doi. org/10.1603/0022-0493-94.6.1419

Nankinga, C.M., Isabirye, B.E., Muyinza, H., Rwomushana, I., Stevenson, P.C., Mayamba, A., Aool W. and Akol, A.M., 2014. Fruit fly infestation in mango: A threat to the Horticultural sector in Uganda. Uganda J. agric. Sci., 15: 1-14.

Navarro-Llopis, V., Alfaro, F., Nguez, J.D., Sanchis, J. and Primo1, J., 2008. Evaluation of traps and lures for mass trapping of Mediterranean fruit fly in citrus groves. J. econ. Ent., 101: 126-131.

Oke, O.A., 2008. Effectiveness of two insecticides to control melon fruit fly (Bactrocera cucurbitae) in cucumber. Eur. J. scient. Res., 22: 84-86.

Pandey, R.R., 2004. Plan your sudex borders for melon fly control. HAW-FLYPM Newsl. (ed. C. Hiraki), Coop. Ext. Serv., Col. Trop. Agric. Human Resour., Univ. Hawaii, February 2004. on world wide web at http://www.fruitfly.hawaii.edu/

Pinero, J.C., Mau, R.F.L., McQuate, G.T. and Vargas, R.I., 2009. Novel bait stations for attract-and kill of pestiferous fruit flies. Ent. Exp. appl., 133: 208-216. https://doi.org/10.1111/j.1570-7458.2009.00912.x

Purcell, M.F., Stark, J.D. and Messing, R.H., 1994. Insecticide effects on three tephridid fruit flies and associated braconid parasitoids in Hawaii. $J$. econ. Ent., 87: 1455-1462. https://doi.org/10.1093/ jee/87.6.1455

Raveen, R., Kamakshi, K.T., Deepa, M., Arivoli, S. and Samuel, T., 2014. Larvicidal activity of Nerium oleander L. flower extracts against Culex quinquefasciatus S (Diptera: Culicidae). Int. J. Mosquito Res., 1: 36-40.

Raveen, R., Pandeeswari, M., Ahmed, F., Reegan, D., Tennyson, S., Arivoli, S. and Jayakumar, M., 2017. Bioefficacy of Nerium oleander L. floral extracts on the larva of three vectors mosquitoes of medical importance. Int. J. Mosquito Res., 4: 65-77.

Ravikumar, C.H., 2005. Studies on fruit flies in guava and mango orchards with special reference to their management through mass trapping. M.Sc. thesis, University of Agricultural Sciences, Dharwad, pp. 90.

Sarada, G., Maheswari, T.U. and Purushotham, K., 2001. Seasonal incidence and population fluctuation of fruit flies in mango and guava. Indian J. Ent., 63: 272-276.

Sarwar, M., 2013. Integrated pest management (IPM). A constructive utensil to manage plant fatalities. Res. Rev. J. agric. Alli. Sci., 2: 1-4.

Sarwar, M., 2014. Some insect pests (Arthropoda: 
Insecta) of summer vegetables, their identification, occurrence, damage and adoption of management practices. Int. J. Sust. Agric. Res., 1: 108-117.

SAS Institute., 2003. SAS 9.1. SAS for windows. Cary, North Carolina.

Selvaraj, P., Sithanantham S., Boopathi T. and Vasumathi S., 2006. Native fruit fly species composition monitoring with methyl eugenol traps. In: International Conference on Biodiversity of Insects: Challenging Issues in Management and Conservation, Bharathiar University, Coimbatore, Tamil Nadu, India. pp. 83.

Singh, M., Gupta, D., Gupta, R. and Kashyap, S.D., 2007. Population dynamics of fruit flies, Bactrocera spp. (Diptera: Tephritidae). Himachal J. agric. Res., 33: 292-294.

Soam, P.S., Singh, T. and Vijayvergia, R., 2013. Citrullus colocynthis (L) and Luff acutangula (L). Source of bioinsecticides and their contribution in managing climate change. Int. J. appl. Biol. Pharma. Technol., 4: 7-9.

Solangi, B.K., Sultana, R., Wagan, M.S. and Ahmed, N., 2011. Repellent action of botanical pesticides against fruit fly, Bactrocera zonata $\mathrm{S}$ in laboratory. Pak. J. Ent. Karachi, 26: 41 -45.

STAT-SOFT, 1998. STATISTICA for windows (computer program electronic Manuel). Tulsa, Ok: Statsoft Inc. Stonehouse, Mahmood, J., Poswal, R.A., Mumford, J., Baloch, K.N. and Chaudhary, Z.M., 2002. Farm field assessments of fruit flies (Diptera: Tephritidae) in Pakistan: distribution, damage and control. $J$. Crop Protec., 21: 661-669. https://doi.org/10.1016/ S0261-2194(02)00018-2

Vargas, R.I. and Prokopy, R., 2006. Attraction and feeding responses of melon flies and oriental fruit flies (Diptera: Tephritidae) to various protein baits with and without toxicants. Proc. Hawaiian entomol. Soc., 38: 49-60.

Vargas, R.I., Peck, S.L., McQuate, G.T., Jackson, C.G., Stark, J.D. and Armstrong, J.W., 2001. Potential for area wide integrated management of Mediterranean fruit by with a braconid parasitoid and a novel bait spray. J. econ. Ent., 94: 817-825. https://doi. org/10.1603/0022-0493-94.4.817

White, I.M. and Elson-Harris, M.M., 1994. Fruit flies of economic significant; their identification and bionomics. CAB International and Aciar, Wallingford.

Zaheeruddin, M., 2007. Study of diffusion and adoption of male annihilation technique. Int. J. Educat. Develop. Inform. Communi. Technol., 3: 89-99. 